\title{
Chronic Inflammation and Carcinogenesis
}

\author{
Pezhman Alavinejad ${ }^{1}$ and Amin Sabbaghan ${ }^{2}$ \\ ${ }^{1}$ Alimentary Tract Research Center, Ahvaz Jundishapur University of Medical Sciences, Ahvaz, Iran \\ ${ }^{2}$ GI Fellowship, Ahvaz Imam Hospital, Ahvaz Jundishapur University of Medical Sciences, Ahvaz, Iran \\ *Corresponding Author: Pezhman Alavinejad, Associate professor of Gastroenterology and Hepatology, Ahvaz Jundishapur University \\ of Medical Sciences, Ahvaz, Iran.
}

Received: June 21, 2019; Published: July 26, 2019

DOI: $10.31080 /$ ASGIS.2019.02.0066

\section{Introduction}

As an approved concept, chronic accumulation of leukocytes and inflammatory mediators in background of chronic inflammation, predispose susceptible cell to mal genesis and neoplastic transformation [1,2]. This condition occurs when the generation of free radicals and active intermediates in an organ exceeds the system's ability to neutralize and eliminate them [3]. Chronic inflammation could result from a variety of factors including bacterial, viral and/or parasitic infections, chemical irritants and non-digestible particles. The longer the inflammation persists, the higher the risk of associated carcinogenesis $[4,5]$.

Several inflammatory mediators such as TNF- $\alpha$, IL-6, TGF- $\beta$, and IL-10 have been shown to participate in both the initiation and progression of cancer [6]. In this regard, measurement of serum acute phase reactant (APR) levels is useful because their abnormalities generally reflect the presence and intensity of an inflammatory process. However, APR measurements in clinical practice are not specific to any particular disease, nor can they distinguish infection from other causes of acute and/or chronic inflammation [7]. The most widely used indicators of the acute phase response are the erythrocyte sedimentation rate (ESR) and C-reactive protein (CRP) levels. The causes of elevation of these indicators include systemic and localized inflammatory and infectious diseases, malignant neoplasms, tissue injury/ischemia and trauma [8,9]. Measurement of APRs may be helpful in assessing the prognosis in some patients with malignancy, assessing the presence or absence of tumor recurrence and distinguishing a clonal growth from a reactive process [10]. This concept further emphasizes the importance of cause and treating chronic inflammation that is predisposing factor for large number of poor outcome conditions including GI malignancies [11].

We should highlight the road map for young researchers to put step in the way, which would result in further determination of exact role of each mediator and inflammatory process and human capability to control many poor conditions to achieve a better future as harvest.

\section{Bibliography}

1. Aggarwal BB., et al. "Targeting inflammatory pathways for prevention and therapy of cancer: short-term friend, longterm foe". Clinical cancer research 15.2 (2009): 425-430.

2. Pezhman Alavinejad and Morteza Nayebi. "Inflammation in Gastrointestinal Tract and Its Importance”. Acta Scientific Gastrointestinal Disorders 2.5 (2019): 01-02.

3. Hanahan D and Weinberg RA. "The hallmarks of cancer". Cell 100.1 (2000): 57-70.

4. Bartsch H and Nair J. "Chronic inflammation and oxidative stress in the genesis and perpetuation of cancer: role of lipid peroxidation, DNA damage, and repair". Langenbeck's Archives of Surgery 391.5 (2006): 499-510.

5. Aggarwal BB., et al. "Inflammation and cancer: how hot is the link?". Biochemical pharmacology 72.11 (2006): 1605-1621.

6. Landskron G., et al. "Chronic inflammation and cytokines in the tumor microenvironment". Journal of Immunology Research (2014).

7. Markanday A. "Acute phase reactants in infections: evidencebased review and a guide for clinicians". In Open forum infectious diseases 2.3 (2015).

8. Kushner I. Acute phase reactants. UpToDate [Internet]. Waltam, MA. (2015).

9. Kisilevsky R and Manley PN. "Acute-phase serum amyloid A: perspectives on its physiological and pathological roles". Amyloid 19.1 (2012): 5-14.

10. Wu Y., et al. "C-reactive protein and inflammation: conformational changes affect function". Biological chemistry 396.11 (2015): 1181-1197.

11. Perkins DJ., et al. "Epigenetic mechanisms governing innate inflammatory responses". Journal of Interferon and Cytokine Research 36.7 (2016): 454-461.

\section{Volume 2 Issue 6 August 2019 \\ (C) All rights are reserved by Pezhman Alavinejad and Amin Sabbaghan.}

